\title{
Short communication: Economic impact among 7 reproductive programs for lactating dairy cows, including a sensitivity analysis of the cost of hormonal treatments
}

\author{
A. Ricci, ${ }^{1}$ M. Li, ${ }^{2} \odot$ P. M. Fricke, ${ }^{2} \odot$ and V. E. Cabrera ${ }^{2 *}$ (이 \\ ${ }^{1}$ Department of Veterinary Science, University of Torino, 10095, Grugliasco, Italy \\ ${ }^{2}$ Department of Dairy Science, University of Wisconsin-Madison, Madison 53705
}

\begin{abstract}
Although hormonal synchronization programs can improve reproductive efficiency of dairy herds, some farmers question the economics of these programs based on the upfront cost of hormonal treatments as opposed to the economic value of the resulting reproductive performance. Our aim was to compare the economic impact of reproductive management programs that incorporate varying degrees of detection of estrus and timed artificial insemination (AI) in dairy herds with year-round calving in confinement total mixed ration systems. A reproductive economic analysis simulation model was used to compare the economic impact of pairs of reproductive management programs. We simulated sets of scenarios for 2 analyses. In the first analysis, we calculated the economic impact of switching from a Presynch-Ovsynch program to a Double-Ovsynch program that included a second $\mathrm{PGF}_{2 \alpha}$ treatment during the Breeding-Ovsynch portion of the program (DoubleOvsynch+PGF). In the second analysis, we conducted a break-even analysis in which the cost of hormonal treatments was incrementally increased within various reproductive management programs. Our analyses revealed that a Double-Ovsynch+PGF program, the most intensive program evaluated, was more profitable than other programs compared, including a PresynchOvsynch program with $100 \%$ timed AI or a PresynchOvsynch program that incorporated detection of estrus, despite the higher upfront cost incurred by using more hormonal treatments. This advantage remained until the cost of hormones was increased 5 to 14 times current US market prices and 2 to 6 times current European market prices. The cost of GnRH had a greater impact on net profit gain than the cost of $\mathrm{PGF}_{2 \alpha}$. In conclusion, more intensive reproductive programs that use more hormonal treatments but result in substan-
\end{abstract}

Received September 26, 2019.

Accepted February 3, 2020.

*Corresponding author: vcabrera@wisc.edu tially increased reproductive performance are more profitable than less intensive programs and remain so even if hormone prices are unusually high.

Key words: reproduction, intensive synchronization program, economic impact

\section{Short Communication}

Advances in the understanding of the reproductive physiology of dairy cows have led to the development of management strategies and technologies that improve reproductive performance. Commercial dairy farms are challenged with making the most profitable management decisions among many options and implementing them correctly. Methods for enhancing fertility and breeding efficiency include detection of estrus (ED) (Xu et al., 1998; Rorie et al., 2002), synchronization of estrus (Folman et al., 1984; Momcilovic et al., 1998), and synchronization of ovulation and timed AI (TAI) (Pursley et al., 1995; Moreira et al., 2001; Souza et al., 2008). The newest TAI programs for first AI (i.e., Double-Ovsynch and G6G) not only increase the AI service rate but also increase conception rate or pregnancy per AI (P/AI) to TAI (Carvalho et al., 2018). Strategies that maximize ED, which are widely implemented at dairies, have made significant contributions to the profitability of dairy herds (Pecsok et al., 1994). A major limitation of ED, however, is the presence of anovular cows, ranging from 5 to $40 \%$ among herds, at the end of the voluntary waiting period (Walsh et al., 2007; Santos et al., 2009; Bamber et al., 2009). The lack of estrous behavior in anovular cows precludes AI to a detected estrus, and many anovular cows are submitted to hormonal programs for TAI.

Hormonal programs for synchronization of ovulation use sequential treatments of $\mathrm{GnRH}$ and $\mathrm{PGF}_{2 \alpha}$ to control follicular development, luteal regression, and time of ovulation (Carvalho et al., 2018). Synchronization of ovulation allows for more precise timing of AI than relying on ED for timing of AI (Pursley et al., 1995; Souza et al., 2008; Valenza et al., 2012; Fricke 
et al., 2014). Furthermore, optimization of the hormonal milieu during the Ovsynch program increases P/ AI for cows at first TAI (Souza et al., 2008; Carvalho et al., 2014) and for cows resynchronized to receive subsequent TAI (Giordano et al., 2012; Lopes et al., 2013; Carvalho et al., 2015). Many farms combine ED (i.e., observation or automated activity monitoring, AAM) and TAI by observing and inseminating cows detected in estrus after the second $\mathrm{PGF}_{2 \alpha}$ treatment of a Presynch-Ovsynch program (Stangaferro et al., 2018, 2019), whereas cows not detected in estrus complete the program and receive TAI. Although inseminating cows detected in estrus during a Presynch-Ovsynch program increases AI service rate, it also decreases $\mathrm{P}$ / AI compared with a Presynch-Ovsynch program without ED (Fricke et al., 2014; Borchardt et al., 2016). This delicate tradeoff depends on the specific reproductive program and the herd's reproductive performance. By contrast, submission of lactating Holstein cows to a Double-Ovsynch+PGF program and TAI for first insemination increased the percentage of cows inseminated within $7 \mathrm{~d}$ after the end of the voluntary waiting period and increased P/AI at 33 and $63 \mathrm{~d}$ after first insemination, resulting in 64 and $58 \%$ more pregnant cows, respectively, compared with submission of cows for first AI after ED at a similar DIM range (Santos et al., 2017). Although primiparous and multiparous cows could have different favorable programs with customized voluntary waiting periods (Stangaferro et al., 2019), most modern dairy farm systems apply a consistent reproductive program to the entire herd for practical management reasons.

The assessment of the overall economic value of various reproductive management programs (Cabrera, 2014; Cabrera and Giordano, 2019) can be achieved by simulating reproductive performance along with the associated costs and benefits on a farm-by-farm basis (Giordano et al., 2011, 2012; Kalantari and Cabrera, 2012) and calculating the expected net return (De Vries et al., 2010; Fricke et al., 2010; Cabrera, 2011). In this study, we used a simulation model to compare the economic impact of current and alternative reproductive programs for managing reproduction on dairy farms with year-round calving that likely have confinement TMR systems (the UW-Cornell DairyRepro\$ decision tool; Giordano et al., 2012). The most novel aspect of our simulation is the sensitivity analysis of hormonal treatment costs among the various reproductive management programs. This study had 2 major objectives: (1) to analyze the profitability of an intensive reproductive program involving more injections (i.e., Double Ovsynch) compared with less intensive ones (PresynchOvsynch) in different geographic areas (i.e., the United States and Europe) depending on variation of hormonal treatment costs, and (2) to estimate how high the cost of hormonal treatments would have to be to render intensive synchronization programs unprofitable.

To answer these questions, we simulated a 1,000-cow commercial dairy herd with year-round calving, using the UW-Cornell DairyRepro\$ decision tool (Giordano et al., 2012). For comparison consistency and to avoid analysis bias, variables that were not studied, such as mortality rate, body weight, involuntary culling rate, lactation curves, milk price, cost of feeding, dry period, fixed cost, female calf values, heifer replacement value, and salvage value, were kept constant among scenarios using default values described by Giordano et al. (2012). Furthermore, we did not evaluate the effect of program compliance on reproductive performance, which can be an issue on some farms (Galvão et al., 2013). Although more complex programs require more compliance, farms using those programs are likely to have a high level of compliance.

Seven reproductive management programs were simulated (Figure 1). Cows were submitted to a PresynchOvsynch program with $100 \%$ TAI, or with service rate by ED before or post TAI. Furthermore, an AAM system and a Double-Ovsynch with double $\mathrm{PGF}_{2 \alpha}$ were simulated. The voluntary waiting period was set at 50 d for programs with ED before the first TAI. The first injection of the synchronization programs occurred at 36 DIM; therefore, the TAI occurred at d 63 for the Double-Ovsynch program and at d 72 for the PresynchOvsynch program. In all cases, milk price was set at $\$ 0.36 / \mathrm{kg}$. The first program simulated a PresynchOvsynch program with ED incorporated after the second $\mathrm{PGF}_{2 \alpha}$ treatment (Presynch-Ovsynch + ED) using TAI for first service for cows not bred to ED (Figure 1A). The second program simulated a PresynchOvsynch program for 100\% TAI, with ED incorporated after first TAI (Presynch-Ovsynch+EDpost; Figure 1B). In programs 1 and 2, P/AI for Presynch-Ovsynch was set at $35 \%$ (Caraviello et al., 2006), service rate for ED was set at $60 \%$, and P/AI for ED was set at $30 \%$ (Giordano et al., 2012). Programs 3 through 5 simulated Presynch-Ovsynch programs (Figure 1C) for 100\% TAI and were simulated with varying $\mathrm{P} / \mathrm{AI}$ to TAI (35\% [baseline], 40\%, and 45\%; Caraviello et al., 2006; Souza et al., 2008; Stangaferro et al., 2018). The sixth program simulated ED with AAM before and after first insemination, with service rate set at $70 \%$ and $\mathrm{P} / \mathrm{AI}$ set at $35 \%$ before first $\mathrm{AI}$ and at $30 \%$ for subsequent $\mathrm{AI}$ (Figure 1D). The cost of the AAM system was included in the simulation (Fricke et al., 2014). The seventh program simulated a Double-Ovsynch+PGF program (Carvalho et al., 2015; Wiltbank et al., 2015) in which P/AI was set at 50\% (Souza et al., 2008, 2013; Santos et al., 2017) (Figure 1E). In all simulations, Ovsynch was 


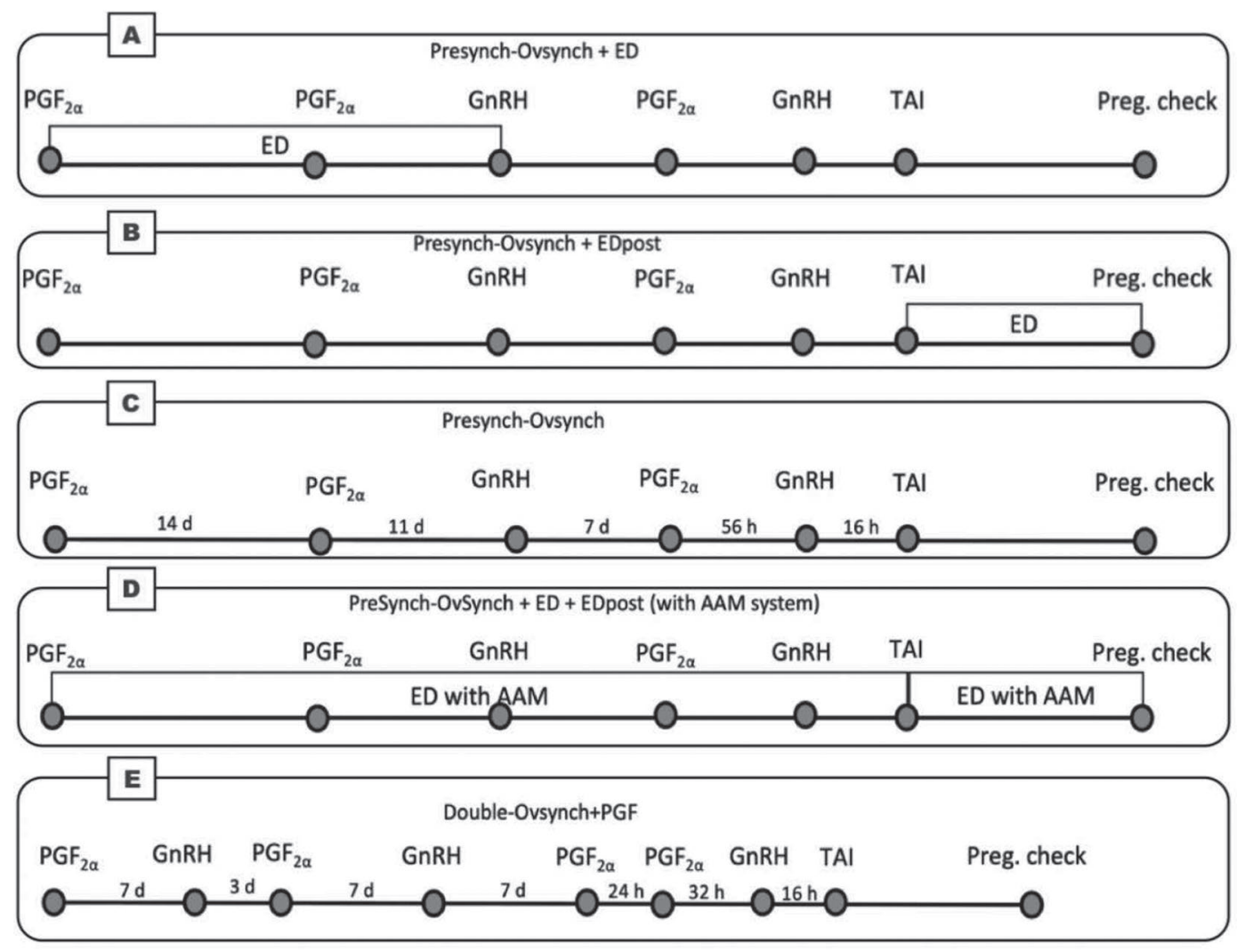

Figure 1. Schematic diagram of treatments used in this study. (A) Presynch-Ovsynch+ED; (B) Presynch-Ovsynch+EDpost; (C) PresynchOvsynch; (D) Presynch-Ovsynch+ED+EDpost (with AAM system); and (E) Double-Ovsynch+PGF. ED = estrus detection; TAI = timed AI; EDpost $=$ estrus detection performed after first AI; AAM = automated activity monitoring; Preg. = pregnancy.

used as the resynch program, with a $30 \% \mathrm{P} / \mathrm{AI}$ to TAI (Lopes et al., 2013). These programs were chosen to reveal the difference in profitability between the most intensive program (i.e., a Double-Ovsynch+PGF program) compared with the most widely used PresynchOvsynch program. The programs that incorporated ED were selected to understand if incorporation of AI to a detected estrus during these programs would be profitable if performed with Presynch-Ovsynch, and this profitability difference was calculated compared with a Double-Ovsynch+PGF program.

Because our first objective was to compare the difference in hormonal treatment costs among the programs, all nonhormonal reproduction costs (e.g., semen, labor for heat detection, pregnancy check) were set at $\$ 0$ in the UW-Cornell DairyRepro\$ decision tool. Involuntary culling and other economic calculations are internally performed by the model and therefore affected by the reproductive performance of each program. The cost of each GnRH treatment was set at $\$ 2.6$ and that of each $\mathrm{PGF}_{2 \alpha}$ treatment was set to $\$ 2.3$ to reflect cost of hormones in the US market (Giordano et al., 2012). To reflect costs in the European market, the cost of each GnRH treatment was set at $\$ 6.7$ and that of each $\mathrm{PGF}_{2 \alpha}$ treatment was set at $\$ 5.1$. The cost of hormonal treatments for the European market was based on average values for the most common commercial $\mathrm{PGF}_{2 \alpha}$ products (cloprostenol $500 \mu \mathrm{g}$ ) and of the most common GnRH (gonadorelin $100 \mu \mathrm{g}$ ) products among the biggest milk producers countries in Europe (Italy, the United Kingdom, and Germany). Based on these costs, the economic simulations were run to calculate the total net profit ( $\$ /$ cow per year) and the aggregated hormone cost of each program. The UW-Cornell DairyRepro\$ decision tool was also used to calculate the number of hormonal treatments required per cow per year for the various programs.

The number of hormonal treatments and net profit gain of the various reproductive management programs were compared using the Presynch-Ovsynch program, with a $35 \% \mathrm{P} / \mathrm{AI}$ as the baseline (Table 1). An economic tradeoff occurred when ED was included in a Presynch-Ovsynch program, which decreased P/AI and increased the service rate (Fricke et al., 2014). This tradeoff was part of our study, and from our results, including ED either before or after TAI would have 
a positive net profit gain over the baseline. Although Presynch-Ovsynch programs use fewer hormonal treatments than a Double-Ovsynch+PGF program (1.4 to 3.0 fewer treatments per cow per year), the Double-Ovsynch + PGF program was more profitable. The Double-Ovsynch+PGF program attained $\$ 42.2$ greater profit per cow per year than the PresynchOvsynch+ED program and $\$ 27.7$ more than PresynchOvsynch+EDpost program based on hormone costs in the US market. Also, the Double-Ovsynch+PGF program was more profitable than the $100 \%$ TAI after a Presynch-Ovsynch program ( $\$ 20.9$ to $\$ 44.3$ more per cow per year, depending on P/AI; Table 1). These results are directly related to the increased reproductive performance of a Double-Ovsynch+PGF program (higher $\mathrm{P} / \mathrm{AI}$ to first $\mathrm{AI}$ ) compared with the other programs. Furthermore, inclusion of ED after the first TAI (i.e., the Presynch-Ovsynch+EDpost program) was a more profitable strategy than using ED either before the first TAI (i.e., the Presynch-Ovsynch+ED program) or not incorporating ED at all based on $35 \%$ and $40 \% \mathrm{P} / \mathrm{AI}$, respectively. The program with AAM could increase the ED rate, which leads to lower use of hormones use when combined with TAI programs, thus offsetting the cost of the system, especially when the hormone price is high. This outcome might be due to fewer cows being submitted to a resynch program at a lower $\mathrm{P} / \mathrm{AI}$ when more intensive programs for first TAI that have an increased P/AI are used. The PresynchOvsynch program with a $45 \% \mathrm{P} / \mathrm{AI}$ had the second greatest net profit among the programs compared because of its higher $\mathrm{P} / \mathrm{AI}$ counteracting the resynch cost for the US market. The gain in profitability when switching to a Double-Ovsynch+PGF program based on US market prices was greater (Table 1) because of the ratio of the costs between $\mathrm{GnRH}$ and $\mathrm{PGF}_{2 \alpha}$, which was $113 \%$ in the US market compared with $131 \%$ in the European market.

The second comparison (second objective) addressed whether a more intensive synchronization program still results in higher profitability than the less intensive reproductive management programs when the costs of hormonal treatments are higher. We therefore conducted sensitivity analyses by incrementally increasing the cost of hormonal treatments to determine the breakeven point at which hormone costs offset the net profit by (1) setting the costs of GnRH and $\mathrm{PGF}_{2 \alpha}$ at US and European market costs and raising both costs by multiples; (2) setting the cost of GnRH at the European market average cost of $\$ 6.7$ and increasing the cost of $\mathrm{PGF}_{2 \alpha}$; and (3) setting the cost of $\mathrm{PGF}_{2 \alpha}$ at European market average cost of $\$ 5.1$ and increasing the cost of GnRH. The ability to vary hormonal treatment costs in the UW-Cornell DairyRepro\$ decision tool made it

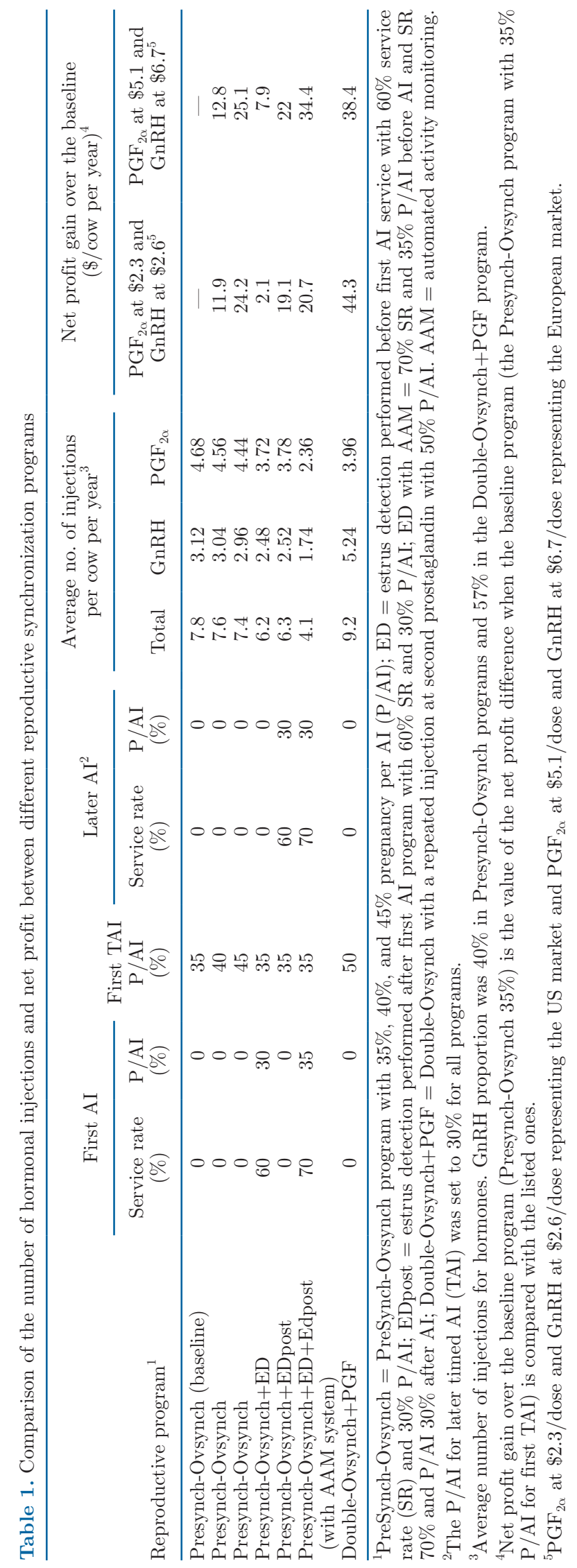


a GnRH price at multiples of the US market (\$/dose)

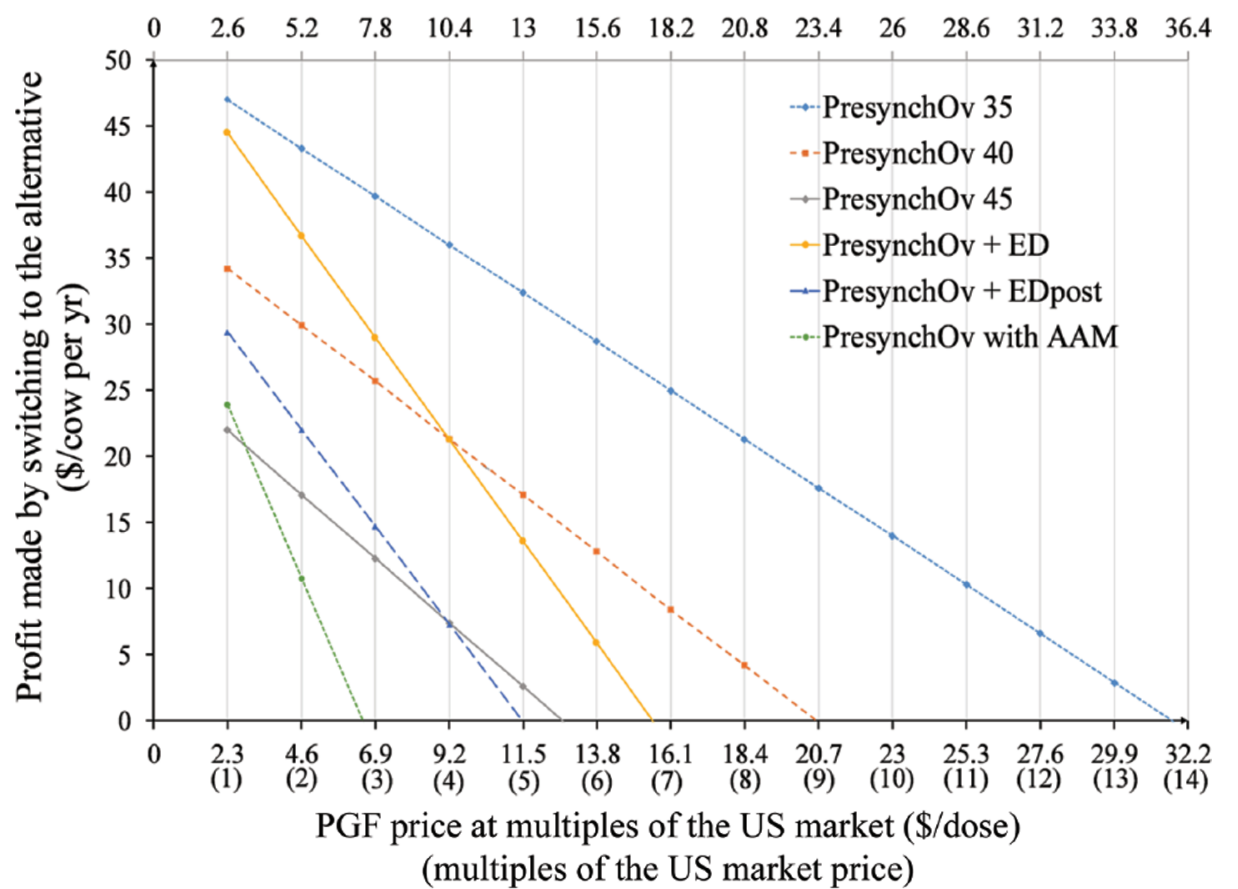

b GnRH price at multiples of the European market (\$/dose)

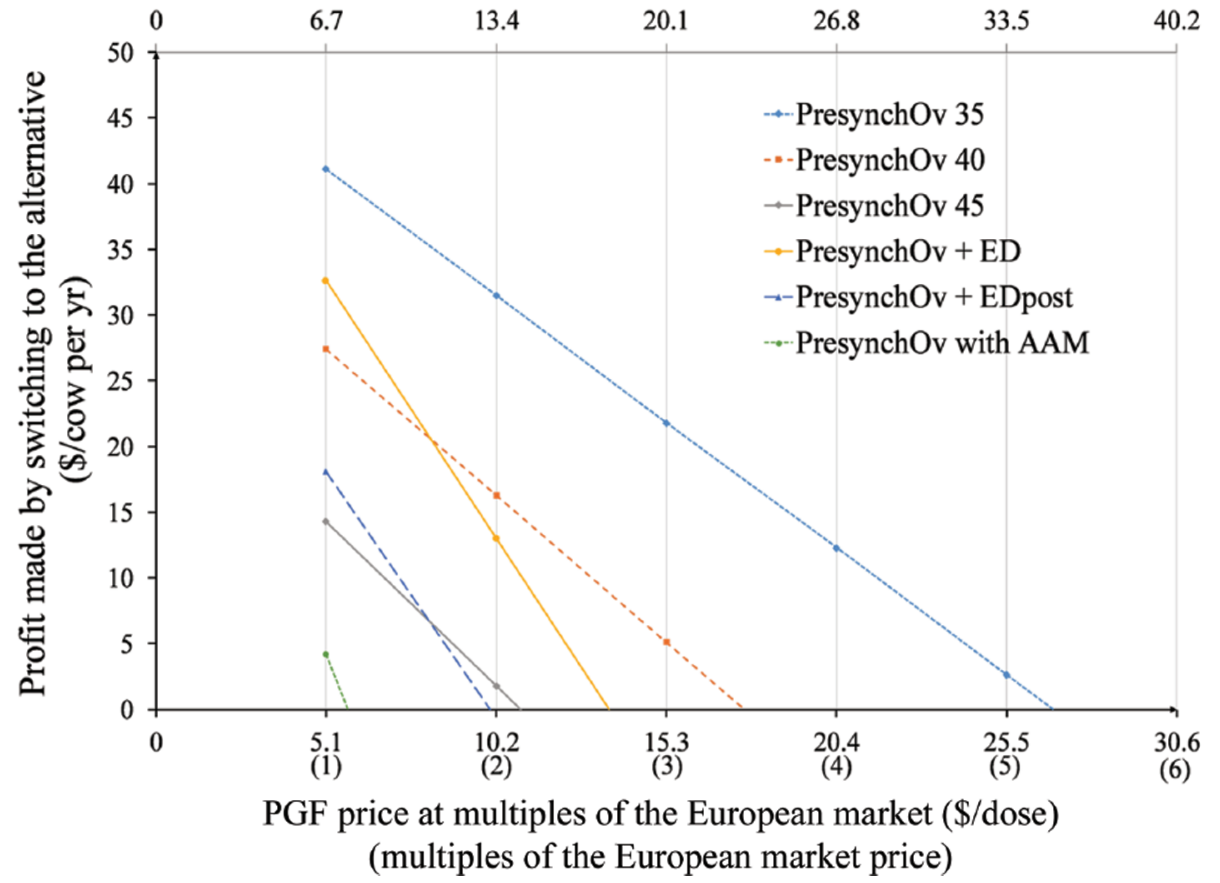

Figure 2. Sensitivity analysis conducted by identifying the break-even points when the net profit gain from switching the Presynch-Ovsynch programs to Double-Ovsynch+PGF program became negative with multiples of GnRH and PGF market prices: GnRH price at multiples of the (a) US market (\$/dose) and (b) European market (\$/dose). PreSynchOv $35=$ Presynch-Ovsynch program with $35 \%$ pregnancy per AI (P/AI); PreSynchOv $40=$ Presynch-Ovsynch program with $40 \%$ P/AI; PreSynchOv $45=$ Presynch-Ovsynch program with $45 \%$ P/AI; PreSynchOv + $\mathrm{ED}=$ Presynch-Ovsynch program with $35 \% \mathrm{P} / \mathrm{AI}+$ estrus detection before first AI service with $60 \%$ service rate and $30 \% \mathrm{P} / \mathrm{AI}: \mathrm{PreSynchOv}+$ EDpost $=$ Presynch-Ovsynch program with $35 \% \mathrm{P} / \mathrm{AI}+$ estrus detection after first AI service with $60 \%$ service rate and $30 \% \mathrm{P} / \mathrm{AI} ; \mathrm{PreSynchOv}$ with $\mathrm{AAM}=$ estrus detection with AAM system before first AI service with $70 \%$ service rate and $35 \%$ P/AI, estrus detection with AAM system after first AI service with $70 \%$ service rate and 30\% P/AI; Double-Ovsynch+PGF = Double-Ovsynch with a repeated injection at second prostaglandin with $50 \% \mathrm{P} / \mathrm{AI}$. AAM = automated activity monitoring. 
possible to simulate these scenarios. For this analysis, the labor cost for administering hormonal treatments was set at $\$ 15 / \mathrm{h}$, labor for transrectal palpation for pregnancy diagnosis was set at $\$ 105 / \mathrm{h}$, and the cost of insemination included semen cost of $\$ 5 / \mathrm{AI}$ and a labor cost of insemination at \$5/AI. All other nonstudied variables, such as lactation curve and other herd parameters, were kept at default values in the UW-Cornell DairyRepro\$ decision tool (Giordano et al., 2012). Briefly, groups of simulations were run to compare the most intensive Double-Ovsynch + PGF program with each of the other defined programs compared in this simulation. In each pair-group comparison, the cost of GnRH, $\mathrm{PGF}_{2 \alpha}$, or both treatments was increased simultaneously by multiples until the net profit gain became negative, and the break-even point was then identified as the intercept of the trend line with the $\mathrm{x}$-axis (Figure 2 ). These $\mathrm{x}$-axis intercepts define the hormonal treatment costs at which a Double-Ovsynch+PGF program would have a net profit equal to that of the program it was compared against.

Based on our analysis, hormonal treatment costs would need to be 5 to 14 times greater in the US market and 2 to 6 times greater in the European market for any of the Presynch-Ovsynch programs to exceed the profitability of the Double-Ovsynch+PGF program. The greater $\mathrm{P} / \mathrm{AI}$ when a Double-Ovsynch $+\mathrm{PGF}$ program for first insemination is used compensates for the additional hormonal treatment costs such that the cost of hormonal treatments would need to increase substantially before the program becomes less profitable than any of the others. When the P/AI of PresynchOvsynch programs increased, the break-even point was reached earlier because the advantage of the increased P/AI of Double-Ovsynch+PGF was decreased.

To investigate the cost of one of the hormonal treatments to reach the break-even point, we ran hypothetical scenarios in which the cost of one hormone was constant but the cost for the other hormone was incrementally increased. In this simulation, the cost of $\mathrm{PGF}_{2 \alpha}$ was set at $\$ 5.1 /$ dose, and the cost of $\mathrm{GnRH}$ was set at $\$ 6.7 /$ dose (i.e., the average European market price). Break-even hormone costs from this analysis are reported in Table 2.

Overall, the break-even cost was more sensitive to the cost of GnRH than to the cost of the $\mathrm{PGF}_{2 \alpha}$ (Table 2). When the cost of GnRH was fixed, the cost of $\mathrm{PGF}_{2 \alpha}$ could increase considerably before the break-even point was reached or no break-even point could be reached by the model. A complete Presynch-Ovsynch program uses $2 \mathrm{GnRH}$ and $3 \mathrm{PGF}_{2 \alpha}$ treatments, whereas the DoubleOvsynch+PGF program uses $4 \mathrm{GnRH}$ and $3 \mathrm{PGF}_{2 \alpha}$ treatments with a higher proportion of $\mathrm{GnRH}$ treatments (40 vs. $57 \%$ ) and a lower proportion of $\mathrm{PGF}_{2 \alpha}$
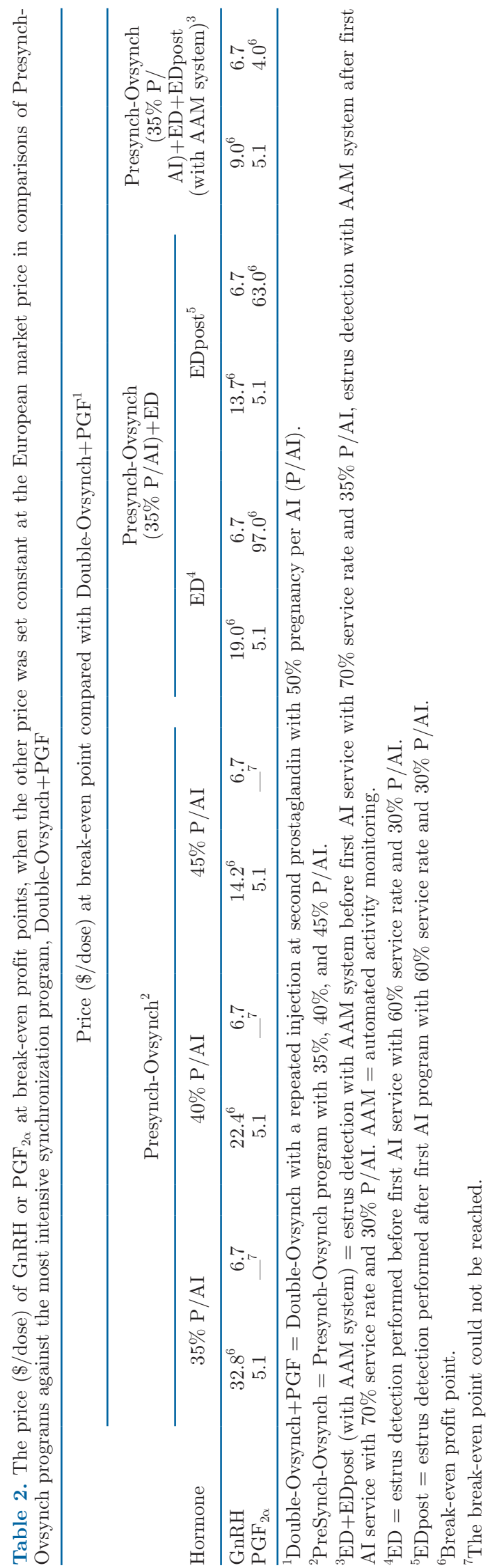
treatments (43 vs. $60 \%$ ). If ED was not incorporated into the reproductive program and the cost of $\mathrm{GnRH}$ was fixed, profit increased even when the cost of $\mathrm{PGF}_{2 \alpha}$ increased. Thus, the break-even points are not reached by the model when the cost of $\mathrm{PGF}_{2 \alpha}$ is increased. For example, compared with Presynch-Ovsynch $40 \% \mathrm{P} / \mathrm{AI}$ with the cost of $\mathrm{PGF}_{2 \alpha}$ fixed at $\$ 5.1 /$ dose, the cost of a GnRH treatment was $\$ 22.4 /$ dose at the breakeven point (Table 2). By contrast, in the first analysis, one break-even point was reached when the price was $\$ 20.7 /$ dose for $\mathrm{PGF}_{2 \alpha}$ and $\$ 23.4 /$ dose for GnRH (Figure 2a, 9 times the US market prices), indicating that a greater $\mathrm{PGF}_{2 \alpha}$ cost determines an even higher cost of $\mathrm{GnRH}$ to reach the break-even point. This outcome can be explained by the higher P/AI of the DoubleOvsynch+PGF program that leads to fewer cows being submitted to a resynch program. Consequently, fewer GnRH treatments per pregnancy are used on average (Table 1). For programs that incorporate ED, fewer cows are submitted to hormonal treatments, which decreases total hormone costs of the program; thus, a break-even point was reached even when the $\mathrm{GnRH}$ cost was fixed, but only when the cost of $\mathrm{PGF}_{2 \alpha}$ was unusually high (Table 2).

In conclusion, more intensive reproductive programs that use more hormonal treatments but result in substantially increased reproductive performance are more profitable than less intensive programs. They remain more profitable even if hormone prices are unusually high, with the exception of efficient AAM systems when hormone costs are high. Results from these analyses could be reproduced or adjusted by applying the UWCornell DairyRepro\$ decision support tool, which is openly available at University of Wisconsin-Madison and Cornell University websites.

\section{ACKNOWLEDGMENTS}

This project was partially supported by USDA National Institute of Food and Agriculture FACT grant (2019-68017-29935; Washington, DC). The authors have not stated any conflicts of interest.

\section{REFERENCES}

Bamber, R. L., G. E. Shook, M. C. Wiltbank, J. E. P. Santos, and P. M. Fricke. 2009. Genetic parameters for anovulation and pregnancy loss in dairy cattle. J. Dairy Sci. 92:5739-5753. https://doi .org/10.3168/jds.2009-2226.

Borchardt, S., P. Haimerl, and W. Heuwieser. 2016. Effect of insemination after estrous detection on pregnancy per artificial insemination and pregnancy loss in a Presynch-Ovsynch protocol: A metaanalysis. J. Dairy Sci. 99:2248-2256. https://doi.org/10.3168/jds .2015-10358.

Cabrera, V. E. 2011. The economic value of changes in 21-day pregnancy rate and what controls this value. In the 21st American Dairy Science Association Discover Conference: Improving Reproductive
Efficiency of Lactating Dairy Cows. Itasca, IL. https://dairymgt .info/publications/abstracts/2011_Discover_Cabrera.pdf.

Cabrera, V. E. 2014. Economics of fertility in high-yielding dairy cows on confined TMR systems. Animal 8(s1):211-221. https://doi.org/ $10.1017 /$ S1751731114000512.

Cabrera, V. E., and J. O. Giordano. 2019. Evaluating the economic value of changing the reproductive management program for a specific dairy farm. DAIReXNET eXtension. Accessed Aug. 16, 2019. https://dairy-cattle.extension.org/evaluating-the-economic -value-of-changing-the-reproductive-management-program-for-a -specific-dairy-farm/.

Caraviello, D. Z., K. A. Weigel, P. M. Fricke, M. C. Wiltbank, M. J. Florent, N. B. Cook, K. V. Nordlund, N. R. Zwald, and C. L. Rawson. 2006. Survey of management practices on reproductive performance of dairy cattle on large US commercial farms. J. Dairy Sci. 89:4723-4735. https://doi.org/10.3168/jds.S0022 $-0302(06) 72522-X$.

Carvalho, P. D., M. J. Fuenzalida, A. Ricci, A. H. Souza, R. V. Barletta, M. C. Wiltbank, and P. M. Fricke. 2015. Modifications to Ovsynch improve fertility during resynchronization: Evaluation of presynchronization with gonadotropin-releasing hormone $6 \mathrm{~d}$ before initiation of Ovsynch and addition of a second prostaglandin $\mathrm{F}_{2 \alpha}$ treatment. J. Dairy Sci. 98:8741-8752. https://doi.org/10 $.3168 /$ jds.2015-9719.

Carvalho, P. D., J. N. Guenther, M. J. Fuenzalida, M. C. Amundson, M. C. Wiltbank, and P. M. Fricke. 2014. Presynchronization using a modified Ovsynch protocol or a single gonadotropin-releasing hormone injection $7 \mathrm{~d}$ before an Ovsynch-56 protocol for submission of lactating dairy cows to first timed artificial insemination. J. Dairy Sci. 97:6305-6315. https://doi.org/10.3168/jds.2014-8222.

Carvalho, P. D., V. G. Santos, J. O. Giordano, M. C. Wiltbank, and P. M. Fricke. 2018. Development of fertility programs to achieve high 21-day pregnancy rates in high-producing dairy cows. Theriogenology 114:165-172. https://doi.org/10.1016/j.theriogenology 2018.03.037.

De Vries, A., J. Van Leeuwen, and W. W. Thatcher. 2010. Economics of improved reproductive performance in dairy cattle. AN156. Department of Animal Sciences, Florida Cooperative Extension Service, Institute of Food and Agricultural Sciences, University of Florida, Gainesville.

Folman, Y., M. Kaim, Z. Herz, and M. Rosenberg. 1984. Reproductive management of dairy cattle based on synchronization of estrous cycles. J. Dairy Sci. 67:153-160. https://doi.org/10.3168/jds .S0022-0302(84)81279-5.

Fricke, P. M., J. O. Giordano, A. Valenza, G. Lopes Jr., M. C. Amundson, and P. D. Carvalho. 2014. Reproductive performance of lactating dairy cows managed for first service using timed artificial insemination with or without detection of estrus using an activitymonitoring system. J. Dairy Sci. 97:2771-2781. https://doi.org/10 $.3168 /$ jds.2013-7366.

Fricke, P. M., S. Stewart, P. Rapnicki, S. Eicker, and M. Overton. 2010. Pregnant vs. open: Getting cows pregnant and the money it makes. eXtension, DAIReXNET Reproduction Resources. https://dairy-cattle.extension.org/pregnant-vs-open-getting-cows -pregnant-and-the-money-it-makes/.

Galvão, K. N., P. Federico, A. De Vries, and G. J. Schuenemann. 2013. Economic comparison of reproductive programs for dairy herds using estrus detection, timed artificial insemination, or a combination. J. Dairy Sci. 96:2681-2693. https://doi.org/10.3168/ jds.2012-5982.

Giordano, J. O., P. M. Fricke, M. C. Wiltbank, and V. E. Cabrera. 2011. An economic decision-making support system for selection of reproductive management programs on dairy farms. J. Dairy Sci. 94:6216-6232. https://doi.org/10.3168/jds.2011-4376.

Giordano, J. O., A. Kalantari, P. M. Fricke, M. C. Wiltbank, and V. E. Cabrera. 2012. A daily herd Markov-chain model to study the reproductive and economic impact of reproductive programs combining timed artificial insemination and estrus detection. J. Dairy Sci. 95:5442-5460. https://doi.org/10.3168/jds.2011-4972.

Kalantari, A. S., and V. E. Cabrera. 2012. The effect of reproductive performance on the dairy cattle herd value assessed by integrating 
a daily dynamic programming model with a daily Markov chain model. J. Dairy Sci. 95:6160-6170. https://doi.org/10.3168/jds .2012-5587.

Lopes, G. Jr., J. O. Giordano, A. Valenza, M. M. Herlihy, J. N. Guenther, M. C. Wiltbank, and P. M. Fricke. 2013. Effect of timing of initiation of resynchronization and presynchronization with gonadotropin-releasing hormone on fertility of resynchronized inseminations in lactating dairy cows. J. Dairy Sci. 96:3788-3798. https:// doi.org/10.3168/jds.2012-6429.

Momcilovic, D., L. F. Archbald, A. Walters, T. Tran, D. Kelbert, C. Risco, and W. W. Thatcher. 1998. Reproductive performance of lactating dairy cows treated with gonadotrophin-releasing hormone $(\mathrm{GnRH})$ and/or prostaglandin $\mathrm{F} 2 \mathrm{a}\left(\mathrm{PGF}_{2 \alpha}\right)$ for synchronization of estrus and ovulation. Theriogenology 50:1131-1139. https: //doi.org/10.1016/S0093-691X(98)00214-3.

Moreira, F., C. Orlandi, C. A. Risco, R. Mattos, F. Lopes, and W. W. Thatcher. 2001. Effects of presynchronization and bovine somatotropin on pregnancy rates to a timed artificial insemination protocol in lactating dairy cows. J. Dairy Sci. 84:1646-1659. https: //doi.org/10.3168/jds.S0022-0302(01)74600-0.

Pecsok, S. R., M. L. McGilliard, and R. L. Nebel. 1994. Conception rates. 1. Derivation and estimates for effects of estrus detection on cow profitability. J. Dairy Sci. 77:3008-3015. https://doi.org/10 .3168/jds.S0022-0302(94)77242-8.

Pursley, J. R., M. O. Mee, and M. C. Wiltbank. 1995. Synchronization of ovulation in dairy cows using $\mathrm{PGF}_{2 \alpha}$ and GnRH. Theriogenology 44:915-923. https://doi.org/10.1016/0093-691X(95)00279-H.

Rorie, R. W., T. R. Bilby, and T. D. Lester. 2002. Application of electronic estrus detection technologies to reproductive management of cattle. Theriogenology 57:137-148. https://doi.org/10.1016/ S0093-691X(01)00663-X.

Santos, J. E. P., H. M. Rutigliano, and M. F. Sá Filho. 2009. Risk factors for resumption of postpartum estrous cycles and embryonic survival in lactating dairy cows. Anim. Reprod. Sci. 110:207-221. https://doi.org/10.1016/j.anireprosci.2008.01.014.

Santos, V. G., P. D. Carvalho, C. Maia, B. Carneiro, A. Valenza, and P. M. Fricke. 2017. Fertility of lactating Holstein cows submitted to a Double-Ovsynch protocol and timed artificial insemination versus artificial insemination after synchronization of estrus at a similar day in milk range. J. Dairy Sci. 100:8507-8517. https://doi .org/10.3168/jds.2017-13210.

Souza, A. H., H. Ayres, R. M. Ferreira, and M. C. Wiltbank. 2008. A new presynchronization system (Double-Ovsynch) increases fertility at first postpartum timed AI in lactating dairy cows. The- riogenology 70:208-215. https://doi.org/10.1016/j.theriogenology 2008.03.014.

Souza, A. H., P. D. Carvalho, R. D. Shaver, M. C. Wiltbank, and V. Cabrera. 2013. Epidemiology of synchronization programs for breeding management in US dairy herds. J. Dairy Sci. 96(Suppl. 1):288.

Stangaferro, M. L., R. Wijma, and J. O. Giordano. 2019. Profitability of dairy cows submitted to the first service with the PresynchOvsynch or Double-Ovsynch protocol and different duration of the voluntary waiting period. J. Dairy Sci. 102:4546-4562. https://doi .org/10.3168/jds.2018-15567.

Stangaferro, M. L., R. Wijma, M. Masello, and J. O. Giordano. 2018. Reproductive performance and herd exit dynamics of lactating dairy cows managed for first service with the Presynch-Ovsynch or Double- Ovsynch protocol and different duration of the voluntary waiting period. J. Dairy Sci. 101:1673-1686. https://doi.org/ $10.3168 /$ jds.2017-13425.

Valenza, A., J. Giordano, G. Lopes Jr., L. Vincenti, M. C. Amundson, and P. Fricke. 2012. Assessment of an accelerometer system for detection of estrus and treatment with gonadotropin-releasing hormone at the time of insemination in lactating dairy cows. J. Dairy Sci. 95:7115-7127. https://doi.org/10.3168/jds.2012-5639.

Walsh, R. B., D. F. Kelton, T. F. Duffield, K. E. Leslie, J. S. Walton, and S. J. LeBlanc. 2007. Prevalence and risk factors for postpartum anovulatory condition in dairy cows. J. Dairy Sci. 90:315-324. https://doi.org/10.3168/jds.S0022-0302(07)72632-2.

Wiltbank, M. C., G. M. Baez, F. Cochrane, R. V. Barletta, C. R. Trayford, and R. T. Joseph. 2015. Effect of a second treatment with prostaglandin $\mathrm{F}_{2 \alpha}$ during the Ovsynch protocol on luteolysis and pregnancy in dairy cows. J. Dairy Sci. 98:8644-8654. https:// doi.org/10.3168/jds.2015-9353.

Xu, Z. Z., D. J. McKnight, R. Vishwanath, C. J. Pitt, and L. J. Burton. 1998. Estrus detection using radiotelemetry or visual observation and tail painting for dairy cows on pasture. J. Dairy Sci. 81:2890-2896. https://doi.org/10.3168/jds.S0022-0302(98)75849 -7 .

\section{ORCIDS}

M. Li ๑ https://orcid.org/0000-0002-0880-8448

P. M. Fricke (으 https://orcid.org/0000-0002-1488-7672

V. E. Cabrera ( https://orcid.org/0000-0003-1739-7457 